\title{
Conocimientos teóricos y prácticos del profesorado sobre reanimación cardiopulmonar y uso del desfibrilador externo automatizado tras un proceso de video-formación Schoolteacher's theoretical and skills knowledge on cardiopulmonary
resuscitation and use of the automated external defibrillator after a video- training process \\ *Rubén Navarro-Paton, *Miguel Cons-Ferreiro, **Vicente Romo-Pérez \\ *U niversidade de Santiago de Compostela (España), **U niversidad deVigo (España)
}

\begin{abstract}
Resumen. El objetivo de esta investigación fue evaluar los conocimientos en Reanimación Cardiopulmonar (RCP) solo manosy utilización del Desfibrilador Externo Automatizado (DEA) del profesorado gallego de Educación Infantil, Primaria y Secundaria tras un proceso de video-formación, comparando el grado del olvido que se produce tras una semana (G 1$)$, al mes (G2) y a los dos meses (G3). Participaron 126 profesores de entre 25-64 años ( $M=44,23 D E=7,74)$, de los cuales $100(79,4 \%)$ eran mujeres y $26(20,6 \%)$ hombres. Los resultados muestran diferencias estadísticamente significativas en la media de respuestas correctas entre el cuestionario inicial y final en todas las dimensiones estudiadas [i.e. Global ( $p<.001$ ); $R C P$ en adulto $(p<.001)$ y $D E A$ y teléfono de emergencias $(p<.001)$ ], entre los grupos $(G 1, G 2$ y $G 3)$, de manera global $(p=.001)$ y conocimiento de parámetros de la RCP ( $p<.001)$. Se encontraron diferencias significativas en la profundidad media en la RCP en adulto (hombres vs mujeres; $p=.046$ ). Respecto a los tiempos de empleo del DEA existen diferencias en aplicar una descarga eficaz entre los grupos G2 y G3 $(p=.028)$, siendo menor el tiempo en el G2. Tras la aplicación de un programa de vídeo-formación se produce un aumento de conocimientos teóricos en el profesorado y se mantiene, al menos, hasta pasados 2 meses con una leve disminución. En cuanto al aprendizaje de las habilidades en RCP sólo manos y aplicación del DEA, se mantienen de igual manera en el tiempo, al menos, hasta los 2 meses.
\end{abstract}

Palabras clave: Profesorado, soporte vital básico, reanimación cardiopulmonar solo manos, desfibrilador externo automatizado, vídeo-formación.

\begin{abstract}
The aim of this research was to evaluate knowledge of hands-only Cardiopulmonary Resuscitation (CPR) and the use of theAutomated External D efibrillator (AED) in G alician schoolteachers of childhood, primary and secondary education, after video-training process, comparing the degree of forgetfulness after one week (G1), one month (G2) and two months (G3). 126 teachers aged 25-64 years ( $M=44.23 \mathrm{SD}=7.74)$ participated, of whom 100 (79.4\%) were women and 26 $(20.6 \%)$ men. The results show statistically significant differences in the mean number of correct answers between the initial and final questionnaire in all the dimensions studied [i.e. Global $(p<.001)$; Adult CPR $(p<.001)$ and AED and emergency telephone $(p<.001)$ ], between the groups $(G 1, G 2$ and $G 3)$, globally $(p=.001)$ and knowledge of CPR parameters ( $p<$ .001). Significant differences were found in the mean depth of only-hands CPR in adults (men vs. women; $p=.046$ ). W ith respect to the time of use of the AED, there are differences in applying an effective discharge between groups $G 2$ and $G 3$ ( $p$ $=.028$ ), with less time in G2. After the application of a training programme there is an increase in theoretical knowledge in the teaching staff and this is maintained at least until after 2 months with a slight decrease. As for the learning of skills in onlyhands CPR and the application of the AED, they are maintained in the same way over time, at least up to two months.
\end{abstract}

Key words: Schoolteacher; basic life support; hands-only cardiopulmonary resuscitation; automated external defibrillator, video-training

\section{Introducción}

Los niños y adolescentes en edad escolar participan diariamente en actividades en las que se producen conductas de riesgo que muchas veces tienen como desen-

Fecha recepción: 07-12-20. Fecha de aceptación: 18-03-21

Ruben Navarro-Paton

ruben.navarro.paton@usc.es lace al gún tipo de lesión física, o incluso lamuerte, como resultado de accidentes como la asfixia. Además de estos accidentes, suelen ocurrir eventos como la muerte súbita cardíaca entre la población escolar, con una incidencia de 3,0-19,7 casos / 100.000 habitantes en países como Estados U nidos (Sakai-Bizmark, et al., 2018), que puede resultar devastador para la familia y la comunidad escolar (Bangall, et al., 2016). Estos sucesos son un problema complejo, donde la mayoría de los casos tie- 
nen un origen cardiaco (Bangall, et al, 2016; M orentin, Suárez-M ier, Aguilera, Arrieta, Audicana, \& FernándezRodríguez, 2012).

En nuestro entorno, el tiempo medio de respuesta de los Servicios de Emergencias M édicas (SEM) es de 8,3 minutos (Sociais-Grespi, Ceniceros-Rozalen, Rubio-Roca, Martinez-Cuellar, García-Sánchez, RipollVera, \& Lesmes-Serrano, 2015) dependiendo del lugar donde estos se localicen, llegando a oscilar entre los 7 y 20 minutos (Claesson, Svensson, Silfverstolpe, \& Herlitz., 2008); por ello, ante una muerte súbita en un centro escolar, el primer interviniente será el profesorado, ya que son los únicos adultos que están en disposición de actuar. Esto muestra la importancia de la formación en primeros auxiliosy SoporteV ital Básico (SVB) que el profesorado debe recibir.

Para poder hacer frente a este tipo de emergencias se debe aplicar el denominado SVB, hasta la llegada de los SEM, que incluye técnicas como la seguridad de la escena, la evaluación de la consciencia, la evaluación de la respiración, la llamada al servicio de emergencias, la reanimación cardiopulmonar (RCP) y la aplicación de un desfibrilador externo automatizado (DEA) (0 lasveengen, et al., 2020). A demás, se sabe que la aplicación del SVB de manera inmediata, cuando una persona sufre una parada cardiaca (PC) extra-hospitalaria, aumenta la tasa de supervivencia(Riva, et al., 2019), ya que la PC es un proceso tiempo dependiente, es decir, su pronóstico puede cambiar ostensiblemente, de forma favorable, si en el menor tiempo posible se comienzan las maniobras básicas hasta la llegada de los equipos de emergencias (CERCP, en línea).

Pero para que esta intervención tenga unas mínimas garantías de éxito, el profesorado de los centros educa tivos debe estar formado en estas maniobras (NavarroPatón, Freire-Tellado, Basanta-Camiño, BarcalaFurelos, Arufe-Giráldez, \& Rodríguez-Fernández, 2018). A pesar de ello, la evidencia científica informa que, tanto los estudiantes universitarios de formación de profesorado (Navarro, Penelas, \& Basanta, 2016), como el profesorado en ejercicio (Gainzta \& Velasco, 2020; NavarroPatón, Cons-Ferreiro, \& Romo-Pérez, 2020a), no tienen la suficiente formación al respecto.

Aunque existen numerosos tipos de formación para la enseñanza de SVB y utilización del DEA (Lago-Ba Ilesteros, Basanta-Camiño, \& Navarro-Patón, 2018; Navarro-Patón, Cons-Ferreiro, M ecías-Calvo, \& RomoPérez, 2021; Navarro, et al., 2020a), de eficacia contrastada (Basanta-Camiño, N avarro-Patón, FreireTellado, BarcalaF Furelos, Pavón-Prieto, Fernández López,
\& N eira-Pájaro, 2017; Navarro-Patón, et al., 2018; 2020a; Pavón-Prieto, Navarro-Patón, Basanta-Camiño, Regueira-M éndez, N eira-Pájaro, \& Freire-Tellado., 2016), los estudios sobre el empleo de estos métodos formativos en el profesorado en nuestro país son limitados.

Por ello, siguiendo las recomendaciones del Consejo Europeo de Resucitación (ERC, Perkins, et al. 2015), que indica que las prácticas de SVB deben ser incluidas en los planes de estudio para capacitar a todos los maestros y profesores, nos proponemos como objetivo de este estudio analizar el efecto de un programa de vídeoformación, sobre el grado de conocimientos teóricos y habilidades prácticas, en materia de SVB (RCP y DEA) adquiridos por el profesorado, a la semana de haberlo recibido, al mes y a los dos meses. Como objetivo secundario se plantea analizar el deterioro de los conocimientos adquiridos a la semana, al mes y a los dos meses en los conocimientosteóricos, en las habilidades prácticas en la aplicación del masaje cardíaco y en la aplicación del DEA. Como hipótesis, se plantean las siguientes: 1. Se produce un aumento en los conocimientos teóricos y prácticos del profesorado tras la video-formación. 2. Se produce un progresivo olvido en los conocimientos y habilidades prácticas en la realización de la RCP y en el uso del DEA. 3. Las habilidades en la real ización de RCP se deterioran antes que la habilidad en el empleo del DEA.

\section{Material y método}

\section{D iseño del estudio}

Se realizó un estudio cuasi-experimental sin grupo control, con seguimiento a la semana, al mes y a los dos meses tras recibir la vídeo-formación.

\section{Participantes}

Se contactó con 178 profesores de Educación Infantil, Primaria y Secundaria de centros públicos de la comunidad autónoma de Galicia. Finalmente, 126 profesores participaron en cada una de las partes del estudio dando su consentimiento para el tratamiento de los datos de la investigación.

\section{Procedimiento}

En primer lugar, se contactó con los centros educa tivos explicando los objetivos de la investigación, solicitando que se remitiese esta información al profesorado del centro. Posteriormente, cuando se recibió la respuesta de los centros educativos, se planificó una re- 
unión informativa donde se le explicó al profesorado el objetivo, la declaración de confidencialidad, el diseño y procedimiento del estudio y su participación volunta ria, así como la posibilidad de retirarse del estudio en el momento que deseasen.

Antes de proceder a realizar la formación se registraron los datos personales de los participantes (edad, sexo, etapa educativa donde impartían docencia y conocimientos previos en SVB) y se les pasó el cuestionario ad hoc de conocimientos previos respecto a la RCP en adultos, secuencia de actuación del DEA y conocimiento del teléfono de emergencias. Posteriormente, se randomizó al profesorado en tres grupos [G1 (evalua ción a la semana de la formación); G2 (evaluación al mes de la formación), G3 (evaluación a los dos meses de la formación)], y se realizó la formación (video-formación y practica de habilidades en RCP y DEA). Posteriormente, se evaluó nuevamente los conocimientos teóricos mediante el mismo cuestionario inicial, las ha bilidades de RCP sólo manos durante dos minutos y la realización de la secuencia de utilización del DEA (figura1).

Durante su participación en el estudio, los sujetos fueron tratados de acuerdo a lo establecido en la Declaración de Helsinki (Asamblea M édica M undial, 2013)

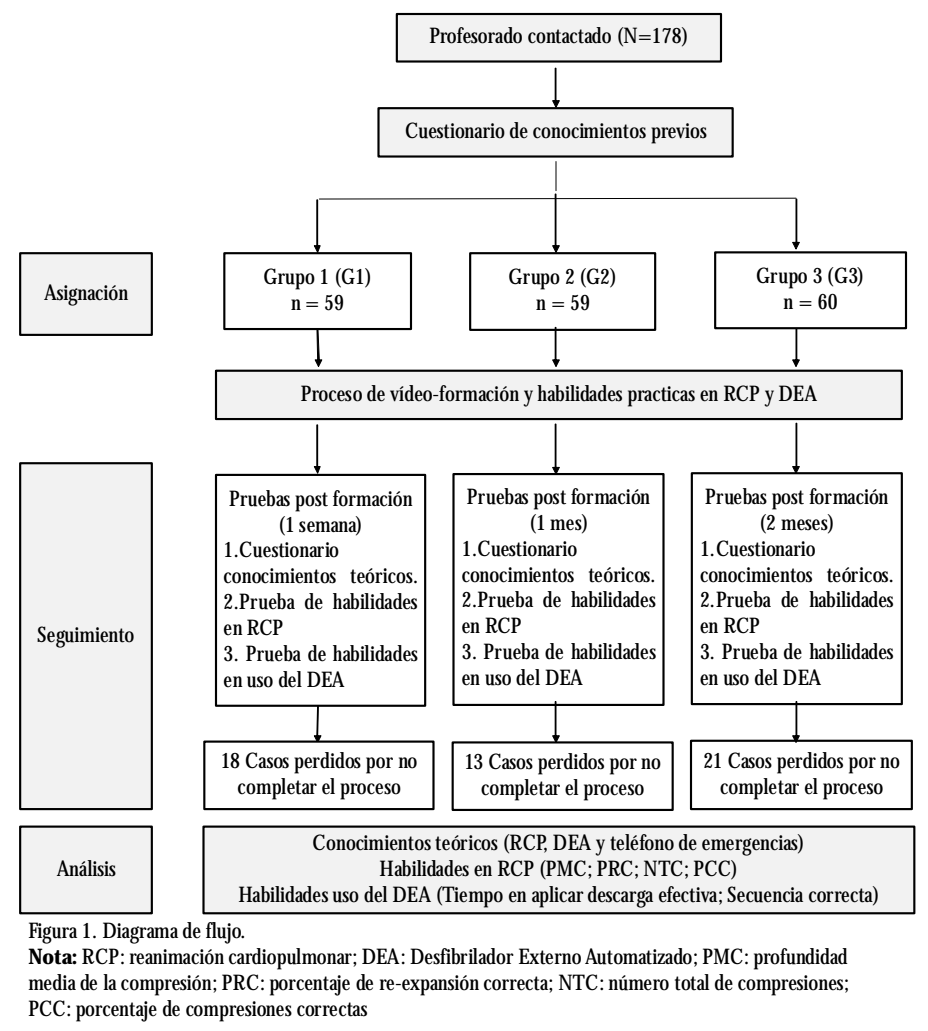

teórica (Conocimiento ratio compresión/ ventilación; compresiones por minuto y profundidad en una RCP en adulto; secuencia de actuación en el uso del DEA y conocimiento del teléfono de emergencias), y por otro, las vincul adas a las habilidades en la ejecución de la RCP (\% compresión correcta; \% re-expansión correcta; número total de compresiones y profundidad media) y del uso del DEA (tiempo en dar una descarga eficaz y secuencia correcta; ver apartado lista de comprobación para uso del DEA). Las variables independientes fueron la edad, género, etapa educativa, años impartiendo docencia y grupo al que se asignó al profesorado.

\section{H erramientas}

\section{Cuestionario}

Para obtener los datos de esta investigación se utilizó un cuestionario estructurado de 12 preguntas utiliza do en otras investigaciones como la de Navarro et al. (2016), Navarro-Patón et al. (2021) o Navarro-Patón et al. (2020a). Las preguntas que se formularon en el cuestionario fueron redactadas en base a seis dimensiones: sociodemográfica (4) (género, edad, etapa en la que imparte docencia, años ejerciendo); formación recibida en primeros auxilios y RCP (2) [formación recibida (dicotómica, sí-no), conocimientos sobre primeros auxilios (escala Likert de 1-5, de ningunosa excelentes)]; conocimientosespecíficos de RCP en adul tos (4), [1 dicotómica (sí-no) y 3 abiertas (ratio compresión ventilación, profundidad de las compresiones y número de compresiones por minuto)]; secuencia de actuación del DEA (1) (abierta) y teléfono de emergencias (1) (Abierta).

\section{Material SoporteVital Básico (SVB)}

El material docente utilizado fue el manual del Consejo Europeo de Resucitación (ERC) en SVB/ DEA, un maniquí Little Anne de Lardal ${ }^{\circledR}$ cada 2 alumnos, un CPRmeter 2 de Laerdal ${ }^{\circledR}$ y un DEA de entrenamien-to cada 6 alumnos.

Lista de comprobación para uso del DEA

En la lista de control para la comprobación del uso del DEA se recogieron las siguientes variables: tiempo de aplicación en descarga eficaz; seguridad en la ejecución (si el profesor no estaba en contacto con el maniquí en el momento de la descarga); orden correcto de ejecución (1. encender, 2. colocar parches, 3. insertar el conector de parches,

\section{Variables de estudio}

Las variables dependientes del estudio fueron, por un lado, las derivadas del cuestionario sobre la parte 
4. Seguir instrucciones, 5. descarga); error que no modifica el objetivo (i.e. Intercambiar el lugar de coloca ción de los parches); O bjetivo de consecución de descarga efectiva ( $\mathrm{N}$ o comete ningún error que altera el objetivo); O bjetivo de calidad (No se comete ningún error, se hace con seguridad y el orden de ejecución es el correcto).

\section{Proceso formativo}

A los participantes se les proporcionaron dos vídeos, uno sobre masaje cardíaco externo sólo manos de 3 minutos y 20 segundos de duración y otro sobre la utilización de DEA de 3 minutos 57 segundos, en el que se simula que un jugador de fútbol-sala sufre una parada cardiaca y es atendido por su entrenador siguiendo los pasos de la cadena de supervivencia. Al final de cada vídeo, un experto resume los puntos clave de la RCP para primeros intervinientes. Los vídeos están disponibles en los siguientes enlaces: masaje cardiaco externo en https:/ / www. youtube.com/ watch?v=ZQ dwoRfTLg; DEA en https:// www. youtube.com/ watch? $=6$ W 4zbqW W Ds. Tras la visualización de los vídeos, los participantes, por parejas, realizaron compresionescardiacas externassobre un maniquí paraadaptarse al muñeco, intercambiándose cada 2 minutos, durante un tiempo total de 6 minutos de compresiones continuas por cada participante. Al finalizar este proceso, a cada grupo de participantes se les entregó un DEA para que simulasen la secuencia de actuación para su aplicación.

\section{Análisis estadístico}

Para el análisis de los datos, las variables cuantitativas se han expresado mediante medidas de tendencia central (medias y desviación estándar). Para el estudio de la asociación entre variables categóricas se usó el estadístico $c^{2}$ de Pearson con el coeficiente de contingencia paralas comparaciones relativas al os conocimientos sobre RCP en adultos, utilización del DEA y teléfono de emergencias en función de la variable G rupo ( $G$ 1; G2 y G3) y el estadístico de Mann W hitney para la comparación por pares entre grupos. Posteriormente, se efectuó un t-test paramuestras relacionadas paracomparar las medias de respuestas correctas antes y después de la formación de manera global, en base al factor RCP y en base factor DEA y teléfono de emergencias. Así mismo, se realizó un análisismultivariante (M AN OVA) para cada variable estudiada respecto a las habilidades en la ejecución de la RCP sólo manos y el tiempo empleado para la aplicación de una descarga efectiva con el
DEA, siendo un primer factor el grupo (G1; G2; G3), y un segundo factor la variable género (hombre-mujer). Se estudiaron los efectos principales y la interacción entre variables, utilizándose el estadístico de Bonferroni para evaluar la significación. Para el tamaño del efecto se empleó el estadístico ç2. Para todo ello, se hizo uso del paquete estadístico IBM SPSS Statistics v. 25.0, estableciendo un intervalo de confianza del $95 \%$ y un nivel de significación de $p>0.05$ en todos los análisis realizados.

\section{Resultados}

En esta investigación se partió de una muestra inicial de 178 profesores, desestimando 52 por no completar todo el procedimiento. La muestra final estuvo constituida por un total de 126 profesores, siendo 100 $(79,4 \%)$ mujeres y $26(20,6 \%)$ hombres con una media de 44,23 (DE $=7,74)$ años. El profesorado impartía docenciaen Educación Infantil ( $n=28 ; 22,2 \%$ ), en Educación Primaria $(n=82 ; 65,0 \%)$ y en Educación Secundaria ( $n=16 ; 12,7 \%$ ) con una media de 15,91 (DE = 8,74 ) años ejerciendo. A pesar de que un total de 71 $(56,3 \%)$ había recibido formación previa en primeros auxilios y SVB, tan sólo $2(1,6 \%)$ indicaron que sus conocimientos eran buenos.

\section{Resultados teóricos antes y después de la vídeo- formación}

En la tabla 1 se pueden observar los datos de aciertos y errores sobre conocimientos adquiridos antes, y tras la vídeo-formación, en cada una de las preguntas formuladas al profesorado. Antes de la formación no existen diferencias sobre el conocimiento entre los tres grupos $(p>.005)$, pero el porcentaje de preguntas correctas después de la formación es significativamente mayor en cada uno de los momentos respecto a las respuestas del cuestionario inicial, en cada uno de los grupos, produciéndose así un aprendizaje con este método formativo $(p<.005)$.

Después de la aplicación del programa de vídeo-formación debemos destacar que se ha encontrado una asociación estadísticamente significativa entre lostres grupos (G1; G2 y G3) cuando se ha valorado el grado del olvido sobre los conocimientos teóricos respecto al ratio de compresión-ventilación $(p=.004)$, en el número de compresiones por minuto $(p<.001)$ y respecto a la profundidad del masaje ( $p=.005$ ).

Si se hace una comparación por pares entre grupos, de los conocimientos teóricos respecto a la RCP, se pro- 


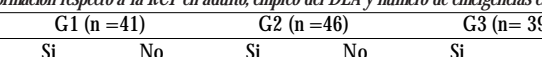

\begin{tabular}{|c|c|c|c|c|c|c|c|c|c|}
\hline & & $\mathrm{Si}$ & No & $\mathrm{Si}$ & No & $\mathrm{Si}$ & No & Chi-cuadrado & Coeficiente de contingencia \\
\hline Sabría & Pre & $\begin{array}{c}6 \\
(14,6 \%)\end{array}$ & $\begin{array}{c}35 \\
(85,4 \%)\end{array}$ & $\begin{array}{c}5 \\
(10,9 \%)\end{array}$ & $\begin{array}{c}41 \\
(89,1 \%)\end{array}$ & $\begin{array}{c}4 \\
(10,3 \%)\end{array}$ & $\begin{array}{c}35 \\
(89,7 \%)\end{array}$ & $.439 ; p=.803$ & $.059 ; p=803$ \\
\hline $\mathrm{RCPe}$ & Post & $\begin{array}{c}35 \\
(14,6 \%)\end{array}$ & $\begin{array}{c}6 \\
(85,4 \%)\end{array}$ & $\begin{array}{c}31 \\
(67,4 \%)\end{array}$ & $\begin{array}{c}15 \\
(32,6 \%)\end{array}$ & $\begin{array}{c}16 \\
(41,0 \%)\end{array}$ & $\begin{array}{c}23 \\
(59,0 \%)\end{array}$ & $17,462: p>.001$ & $.349 ; p>.001$ \\
\hline & & Correcto & Incor recto & Correcto & Incorrecto & Correcto & Incorrecto & Chi-cuadrado & Coeficiente de contingencia \\
\hline
\end{tabular}

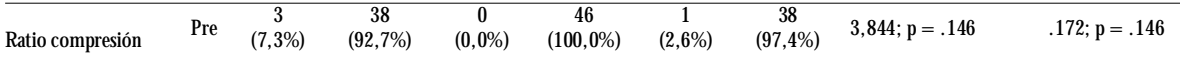

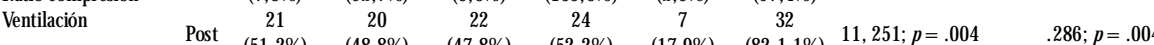

\begin{tabular}{lccccccccc} 
& Post & $(51,2 \%)$ & $(48,8 \%)$ & $(47,8 \%)$ & $(52,2 \%)$ & $(17,9 \%)$ & $(82,1,1 \%)$ & 11,$251 ; p=.004$ & $.286 ; p=.004$ \\
\hline \multirow{2}{*}{ Compresiones por } & \multirow{2}{*}{ Pre } & 2 & 39 & 0 & 46 & 2 & 37 & $2379 ; p=.304$ & $.136 ; p=.304$
\end{tabular}

minuto

\begin{tabular}{lcccc} 
minuto & Post & 34 & 7 & 21 \\
& & $(82,9 \%)$ & $(17,1 \%)$ & $(45,7 \%$ \\
\hline $\begin{array}{l}\text { Profundidad del } \\
\text { masaje }\end{array}$ & Pre & $\begin{array}{c}1 \\
(2,4 \%)\end{array}$ & $\begin{array}{c}40 \\
(97,6 \%)\end{array}$ & $(4,3 \%)$ \\
& Post & 32 & 9 & 24
\end{tabular}

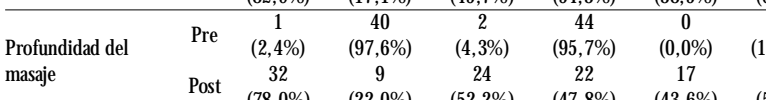

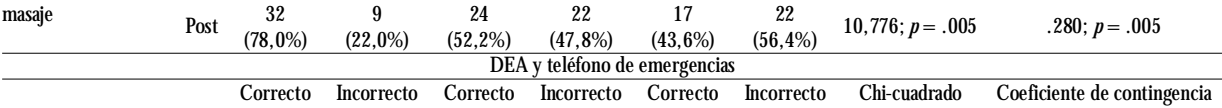

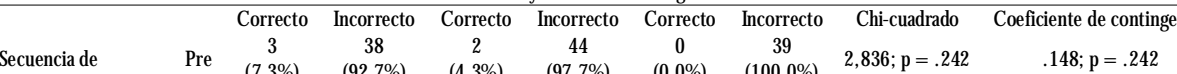

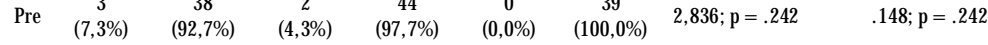

$\begin{array}{lcccccccc}\text { colocación y } & (7,3 \%) & (92,7 \%) & (4,3 \%) & (97,7 \%) & (0,0 \%) & (100,0 \%) & 2,836 ; p=.242 & \\ \text { utilización del DESA } & \text { Post } 17(41,5 \%) & 24 & 16 & 30 & 13 & 26 & .663: p=.718 & .072 ; p=.718\end{array}$

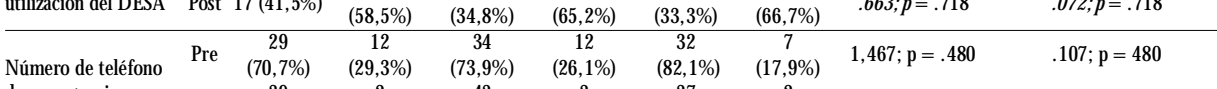

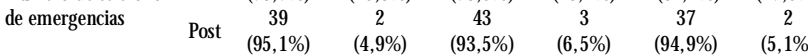

duce una disminución entre el $\mathrm{G} 1$ y el $\mathrm{G} 2$, respecto a las respuestas correctas, en el número compresiones por minuto $(p<.001)$ y en la profundidad del masaje $(p$ $=.012$ ), produciéndose menos respuestas correctas en el $\mathrm{G} 2$.

Si las comparaciones se hacen entre el G1 y el G3, estas diferencias se producen en el ratio de compresión-ventilación ( $p=.002)$, en el número de compresiones por minuto $(p<.001)$ y en la profundidad del masaje ( $p=.002$ ), siendo mayor el número de respuestas correctas en el G1. Si se comparan los grupos $G 2$ y G3, únicamente existen diferencias en el ratio de compresión-ventilación ( $p=.004$ ), siendo mayores las respuestas correctas en el G2. En base a estos resultados podemosindicar que se produce un olvido a medida que pasa el tiempo tras la vídeo-formación ( $G 1$ vs. G2, G1 vs. $G 3$ y $G 2$ vs. G3).

No se han encontrado diferencias estadísticamente significativas en la respuesta de la secuencia de empleo del DEA ( $p=.718)$ ni en el conocimiento del teléfono de emergencias $(p=.936)$. Puede observarse que a medida que pasa el tiempo en relación a la re-evalua ción tras la vídeo-formación, no se produce olvido en el conocimiento teórico de la secuencia de utilización del DEA ni del número de emergencias.

En la figura 1 se pueden observar las puntuaciones globales medias [media (M) y error estándar (ES)] del profesorado respecto a las respuestas correctas antes y después de la formación $(M=.88 ; E S=.06$ vs $M=$ 2,84; $\mathrm{SE}=.13$; $\mathrm{t}(125)=-14,685, p<.001, r=.20)$. En las comparaciones por agrupación de dimensiones, se obtuvieron los siguientes resultados: media de respuestas correctas en la dimensión RCP en adultos entre

mensiones estudiadas. $=.711$ ). el prey el post test $(M=$ .08; $\mathrm{SE}=.03$ vs 1,53 ; $\mathrm{SE}=.10 ; \mathrm{t}(125)=$ $13,699, p<.001, r=$ .21); media de respuestas correctas en la secuencia de utilización del DEA y conocimiento del teléfono de emergencias $(.79 ; \mathrm{SE}=$ .04 vs 1.30; SE $=.04 ; \mathrm{t}$ $(125)=-8,395, p<$ $.001, r=.21)$, produciéndose un aumento en los conocimientos teóricos tras la vídeo-formación en todas las di-

Del mismo modo, en el MANOVA realizado, sólo se han encontrado diferencias estadísticamente significativas entre los tres grupos en la media de respuestas correctas de manera global $(F(2,123)=7,545 ; p=$ $\left..001, c_{2}=.10\right)$, disminuyendo estas medias a medida que pasa más tiempo en la re-evaluación. Lo mismo ocurre en el conocimiento de los parámetros de la RCP en adultos $(F(2,123)=9,965 ; p<.001$, ç $=.14)$, no siendo así en el conocimiento de la secuencia del empleo del DEA y número de teléfono de emergencias ( $p$

\section{$\mathrm{H}$ abilidades respecto a la calidad de la RCP}

En la tabla 2 se muestran las medias y desviaciones

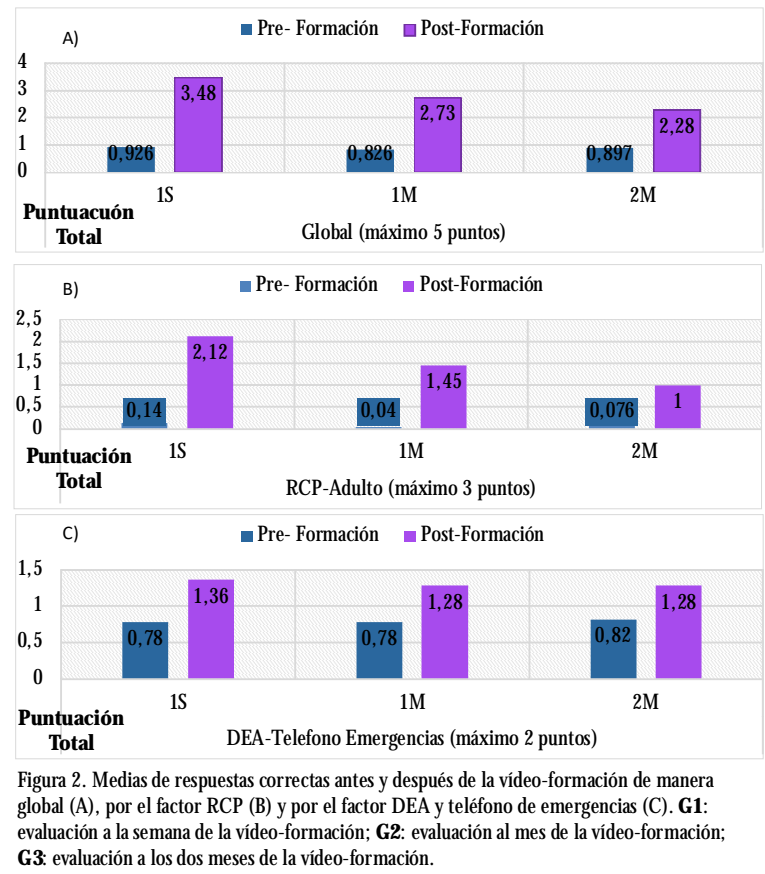


estándar de los parámetros recogidos por el CPRmeter 2 de Laerdal después del programa de vídeo-formación, de manera global y por género.

\begin{tabular}{|c|c|c|c|c|c|c|c|}
\hline \multirow[b]{2}{*}{ Variable } & \multirow[b]{2}{*}{ Grupo } & \multicolumn{2}{|c|}{ Hombres } & \multicolumn{2}{|c|}{ Mujeres } & \multicolumn{2}{|c|}{ Total } \\
\hline & & M & $D E$ & M & $D E$ & M & $\mathrm{DE}$ \\
\hline \multirow{3}{*}{$\begin{array}{l}\text { PMC } \\
(\mathrm{mm})\end{array}$} & G1 & 50,55 & 5,68 & 44,80 & 8,76 & 46,34 & 8,47 \\
\hline & G2 & 46,80 & 8,66 & 42,00 & 8,71 & 43,07 & 8,84 \\
\hline & G3 & 48,40 & 2,70 & 47,09 & 8,88 & 47,27 & 8,30 \\
\hline \multirow{3}{*}{ PRC (\%) } & G1 & 99,91 & 0,30 & 100,0 & 0,0 & 99,98 & 0,15 \\
\hline & G2 & 99,10 & 2,84 & 99,91 & 0,37 & 99,73 & 1,37 \\
\hline & G3 & 99,20 & 1,30 & 96,59 & 9,53 & 96,95 & 8,90 \\
\hline \multirow{3}{*}{$\begin{array}{c}\text { NTC } \\
(2 \mathrm{~min})\end{array}$} & G1 & 231,33 & 47,09 & 235,18 & 47,09 & 232,37 & 45,49 \\
\hline & G2 & 192,60 & 42,68 & 196,91 & 42,68 & 195,96 & 54,53 \\
\hline & G3 & 265,80 & 66,65 & 245,94 & 75,31 & 248,62 & 73,66 \\
\hline & G1 & 47,73 & 42,89 & 24,90 & 39,23 & 31,02 & 40,99 \\
\hline \multirow[t]{2}{*}{ PCC (\%) } & G2 & 46,30 & 35,16 & 41,17 & 42,57 & 42,31 & 40,72 \\
\hline & G3 & 15,60 & 23,62 & 33,53 & 44,1 & 31,11 & 42,14 \\
\hline
\end{tabular}

Los resultadosdel M AN OVA realizado indicaron que existe un efecto principal significativo del factor grupo ( $F=6,738, p=.002 ;$; $2=.060)$, en el número de compresiones totales, siendo superiores en el $\mathrm{G} 2$, pero estando por encima del rango recomendado (100-120 compresiones minuto). Losúnicos que se mantienen en ese rango son los profesores a los que se evaluó a la semana (G1) de la vídeo-formación. Respecto al géne$r o$, se encontraron diferencias estadísticamente significativas en la profundidad media ( $F=4,069, p=.046$; ç2 $=.034$ ), siendo mayor en los hombres que en las mujeres pero sin llegar a la profundidad mínima de 5 $\mathrm{cm}$ en ninguno de los casos. Respecto al resto de factores analizados no se encontraron diferencias estadísticamente significativas entre los factores género, ni grupo ni en la interacción de ambos. Debemos destacar que el porcentaje de re-expansión correcta es casi del $100 \%$ en todos los grupos de manera global y por género. Respecto a la profundidad media tan sólo los hombres del grupo de una semana al canzan el mínimo de profundidad, disminuyendo en el resto a medida que se valora con el paso del tiempo.

En las comparaciones por pares, se encontraron diferencias estadísticamente significativas en el número total de compresiones entre el $\mathrm{G} 1$ y el $\mathrm{G} 2$ (.032), siendo superioresen el $\mathrm{G} 1$, y encontrándose dentro del rango correcto (100-120 compresiones en 1 minuto), y entre el G2 y G3 (.002), siendo superiores en el G3; en este último caso ninguno de los grupos se encuentra dentro del rango correcto.

$H$ abilidades respecto al uso y secuencia del DEA

En la tabla 3 se muestran las medias y desviaciones estándar de los tiempos empleados en aplicar una des- carga eficaz después del programa de vídeo-formación, a la semana ( $G 1)$, al mes (G2) y a los dos meses $(G 3)$ de forma global y por género, de aquel profesorado que fue capaz de conseguir el objetivo [sin errores que lo alterasen (i.e. parches mal colocados o realiza una descarga sin pegar los parches)].

\begin{tabular}{|c|c|c|c|c|c|c|c|}
\hline \multicolumn{2}{|c|}{ Variable } & \multicolumn{2}{|c|}{ Hombres } & \multicolumn{2}{|c|}{ Mujeres } & \multicolumn{2}{|c|}{ Total } \\
\hline Tiempo (S) & Grupo & Media & $D E$ & Media & $D E$ & Media & $D E$ \\
\hline & G1 & 69,00 & 15,49 & 70,87 & 14,58 & 70,37 & 14,66 \\
\hline \multirow[t]{2}{*}{ Tiempo aplicación } & G2 & 77,00 & 15,48 & 76,89 & 19,48 & 75,91 & 18,50 \\
\hline & G3 & 61,60 & 10,45 & 66,69 & 14,33 & 66,00 & 13,86 \\
\hline
\end{tabular}

Los resultados del MAN OVA realizado indicaron que existe un efecto principal significativo del factor grupo $[F(2,120)=4,050 ; p=.020, c 2=.063]$, pero no en el factor género ( $p=.564)$, ni en la interacción entre los factores grupo y género ( $p=.904)$. En la comparación por pares, se evidencian diferencias estadísticas significativas en el tiempo empleado en aplicar una descarga eficaz entre los profesores a los que se les pasa la prueba al mes (G2) y a los dos meses (G3) ( $p=.020)$, siendo menor el tiempo empleado por el profesorado del $\mathrm{G} 3$.

\section{Discusión}

El objetivo de esta investigación fue analizar el efecto de una vídeo-formación sobre el grado de conocimientos teóricos y habilidades prácticas en materia de SVB (RCP y DEA) adquiridos por el profesorado, a la semana de haberlo recibido, al mes y a los dos meses. Los resultados globales obtenidos muestran que, con la formación recibida de menos de 20 minutos (dos vídeos que no superan los 10 minutos entre ambos, 6 minutos de compresiones sólo manos y 2 minutos en la práctica de empleo del DEA), el profesorado participante en esta investigación ha mejorado los conocimientos teóricos en RCP solo manos en adultos, en la secuencia de actuación para el empleo de un DEA y el conocimiento del teléfono de emergencias, demostrando que este método deenseñanza puedemejorar estos conocimientos (Aqel \& Ahmad, 2014; Hernández-Padilla, Suthers, Granero M olina, \& Fernández Sola, 2015; N avarroPatón, et al. , 2021), así como unos resultados en habilidades en la realización de RCP sólo manos y DEA similares a otros realizados en nuestro país (N avarro-Patón, et al. , 2021; N avarro-Patón, et al., 2018).

En base a los resultados obtenidos en la evaluación inicial, aunque el profesorado afirmó haber recibido formación en cuanto a primeros auxilios y SVB, cuando se 
le preguntó por contenidos propios de la RCP y DEA, muy pocos contestan correctamente, siendo mejores los resultados sobre el conocimiento del teléfono de emergencias (Navarro-Patón, et al., 2021). Por ello, debemos indicar que el profesorado participante en este estudio, inicialmente no poseía los suficientes conocimientos en SVB. Este hecho podría dificultar que el profesorado implementase este tipo de contenidos en sus clases, según los currículos educativos actuales (en aquellas materias que así estuviese estipulado), y de este modo contribuir a la formación de escolares de acuerdo con organismos internacionales como laERC (Böttiger, et al., 2016; Lukas, Van Aken, Mölhoff, Weber, Rammert, W id, \& Bohn, 2016; Semeraro, et al., 2017).

Sin embargo, los resultados sobre el conocimiento teórico obtenido en esta investigación, tras la aplicación de la vídeo-formación, sugieren que el empleo de este tipo de formación (vídeos) y la práctica posterior de habilidades, es suficiente para aumentar los conocimientos de RCP y uso del DEA, así como el conocimiento del teléfono de emergencias, resultados parecidos a los obtenidos por Navarro-Patón et al. (2021) en profesorado, por M éndez-Martínez, M artínez-Isasi, GarcíaSuarez, De la Peña-Rodríguez, Gómez-Salgado, \& Fernández-García (2019) en estudiantes de ciencias de la salud y Navarro et al. (2016) y Navarro-Patón et al. (2018) en estudiantes del grado de Educación Primaria.

Respecto a las habilidades en la realización de RCP básica, si comparamos los criterios propuestos en las guías de la ERC (Perkins, et al., 2015; i.e. profundidad de aproximadamente $5 \mathrm{~cm}$, sin sobrepasar los $6 \mathrm{~cm}$, con una frecuencia de 100 a 120 compresiones por minuto, permitiendo que el tórax se re-expanda totalmente en cada compresión realizada), debemos indicar que el profesorado de nuestro estudio no alcanza los parámetros de calidad en cuanto a la profundidad, sólo los hombres del $\mathrm{G} 1$, siendo un poco más cercanosa los estándares en los varones que en las mujeres (Méndez-Martínez, et al., 2019) y mejores en el $G 1$ que en $G 2$ o G3. Se sabe que este parámetro podría ser mejorado mediante un entrenamiento regular (K rasteva, IJ ekova, \& Didon 2011). O curre lo mismo con el porcentaje de compresión correcta que alcanza tan sólo un $50 \%$ en el G1, que disminuye con el paso del tiempo, datos que difieren de los resultados de otros estudios en personal lego, profesorado y en estudiantes universitarios (González-Salva do, Fernández-M éndez, Barcala-Furelos, Peña-Gil, González-J uanatey, \& Rodríguez-Núñez, 2016; Nava rro-Patón, et al., 2021; Navarro-Patón, Freire-Tellado, Mateos-Lorenzo, Basanta-Camiño, Rodríguez-López, \&
Lago-Ballesteros, 2020b; N avarro-Patón, et al., 2018). Respecto al porcentaje de re-expansión correcta, tanto hombres como mujeres, y en los tres grupos de forma global, obtienen valores de más del $90 \%$. Por otro lado, cabe indicar que el profesorado sólo es capaz de realizar una frecuencia correcta en el $\mathrm{G} 1$, estando por debajo de esta los del $G 2$ y por encima los del $G 3$.

Respecto al uso del DEA, la vídeo-formación fue eficaz, con el $100 \%$ de los participantes obteniendo el objetivo en la aplicación de este dispositivo, resultados superiores a los encontrados por M éndez-M artínez et al. , (2019) con estudiantes de enfermería y fisioterapia o los de Basanta-Camiño et al. (2017) o Navarro-Patón et al. (2018) con estudiantes del Grado de Educación Primaria.

Por todo lo anterior, podemos aceptar, en parte, la primera hipótesis planteada ya que se ha producido un aumento en los conocimientosteóricossobre RCP, DEA y teléfono de emergencias y las habilidades en el uso del DEA, pero no en las habilidades de la RCP. Aunque en este último aspecto, los resultados no son adecuados con los estándares de calidad, no deben ser desestima dos ya que con la formación con este tipo de instrumentos y métodos, muy pocos colectivos, como estudiantes de profesorado en formación (Navarro-Patón, et al., 2018) o incluso profesionales dedicados a las emergencias médicas, son capaces de alcanzar y superar estos parámetros (González-Salvado et al., 2016).

Como objetivo secundario en nuestro estudio se planteó analizar el deterioro de los conocimientos adquiridos a la semana, al mes y a los dos meses en los conocimientos teóricos, en las habilidades prácticas en la aplicación del masaje cardíaco y en la aplicación del DEA.

Los resultados, como norma general, muestran que la retención a medio plazo (1-2 meses) de los conocimientos teóricos y habilidades va disminuyendo en lo que respecta a la RCP, no siendo así en el uso del DEA. Esto es similar a la evidencia sobre la formación en SVB en otras investigaciones (Baldi, et al. , 2017; Cortegiani, et al., 2017, N avarro-Patón, et al., 2021; N avarro-Patón, et al., 2018; Nishiyama, et al., 2015). Aún así, la vídeoformación y práctica de habilidades, ha sido suficiente para mejorar los conocimientos y habilidades del profesorado participante y nos hace pensar que estos progra mas pueden ser útiles y factibles, ya que no requieren de costosos medios técnicos ni organizativos e, incluso, con mejores resultados que si el ciudadano sin forma ción es guiado por una central de emergencias (Nava rro-Patón, Freire-Tellado, Pavón-Prieto,Vázquez-López, Neira-Pájaro, \& Lorenzana-Bargueiras, 2017). Por otro 
lado, si tenemos en cuenta que el deterioro de las habilidades en el uso de DEA, si bien es menor que las de la RCP básica, sería recomendable incluir este tipo de formación en todos los cursos de SVB, extendiendo este conocimiento a la mayor población posible, ya que es un elemento fundamental para la supervivencia en los ca sos de una PC con ritmo desfibrilable (en la RCP básica por testigos).

En cuanto al teléfono de emergencias, debemos decir que es más conocido por parte de todo el profesora do (Navarro-Patón, et al., 2021), pudiendo ser debido a las campañas divulgativas en los diferentes medios de comunicación, si bien este conocimiento aumentó tras el proceso de vídeo-formación.

Por todo lo anterior podemos aceptar, en parte, la hipótesis de que se produce un progresivo olvido en los conocimientos y habilidades prácticas en la realización de la RCP, pero no en el uso del DEA. En consecuencia, podemos aceptar la tercera hipótesis en la que se proponía que las habilidades en la realización de RCP se deterioran antes que la habilidad en el empleo del DEA.

Como limitaciones de nuestro estudio, debemos destacar que las habilidades tanto en RCP básica como en el empleo del DEA se realizaron mediante una simulación, por lo que no sabemos cómo podrá reaccionar el profesorado ante una situación real. Por otro lado, aunque la participación fue voluntaria, debemos tener en cuenta que la motivación hacia el programa de vídeoformación sobre SVB de algunos de los participantes pudo influir en el desarrollo del estudio. En nuestro estudio no se emplearon dispositivos que proporcionan retroalimentación inmediata en el masaje cardiaco, lo que pudo influir en la calidad del mismo. Finalmente, debemos tomar con cautela los resultados del estudio, sobre todo en las habilidades de la RCP, debido la asimetría de los grupos, con una clara prevalencia del género femenino, por lo que se deben tomar con cierta cautela las conclusiones de las comparaciones entre géneros.

\section{Conclusiones}

El profesorado de esta investigación no poseía conocimientos basales suficientes en materia de SVB (RCP y DEA), no siendo así en el conocimiento del teléfono de emergencias, aún indicando que habían recibido formación previa.

Tras la aplicación de la vídeo-formación para la enseñanza de SVB, se produce un aumento de conocimientos teóricos en el profesorado que se mantiene al me- nos hasta pasados 2 meses (G3), aunque se produce una ligera pérdida de conocimientos del $\mathrm{G} 1$ a $\mathrm{G} 2$ y de $\mathrm{G} 2$ a G3.

Respecto a las habilidades de la RCP, la vídeo-formación junto con la práctica de 6 minutos, produce un aumento en las habilidades que sólo llegan a los parámetros recomendados en el ritmo de compresiones en el G1 y en el porcentaje de re-expansión correcta, que se mantiene a lo largo de los 2 meses (G3) y en la profundidad media en los hombres del grupo del G1.

Sobre el aprendizaje del uso del DEA, se ha producido una mejora significativa que se mantiene al cabo de los 2 meses, al igual que en el conocimiento del teléfono de emergencias. Por todo ello, un programa de vídeoformación y práctica de habilidades de estas características puede ser factible y viable para la enseñanza de SVB a profesorado en activo.

Como propuestas de formación indicamos que, 1) los entrenamientos en habilidades de RCP deben ser real izadas con retroalimentación en tiempo real, lo que ayudaría a mejorar la calidad del masaje cardíaco; 2) EI entrenamiento o refresco respecto al conocimiento teórico y las habilidades del masaje cardiaco deben realizarse con una periodicidad no superior a 2 meses; 3 ) el entrenamiento o refresco en el uso del DEA, puede real izarse pasados los 2 meses, ya que el deterioro en el conocimiento teórico y habilidades permanecen durante más tiempo que las habilidades del masaje cardíaco.

\section{Referencias}

Aqel,A.A., \&Ahmad, M.M., (2014). Highfidelity simulation effects on CPR knowledge, skills, acquisition, and retention in nursing students. Worl dviews Evid. Based N urs, 11, 394-400. doi:10.1111/ wvn. 12063

Asamblea Médica Mundia (2013). Dedaración de Helsinki de la AMM - Principios éticos para las investigaciones médicas en seres humanos. Recuperado de https:/ / www. wmanet/ es/ policies-post/ declaracion-de-helsinki-de-larammprincipios-eticos-paralas-investigaciones-medicas-enseres-humanos/

Bagnall, R. D., Weintraub, R. G., Ingles, J., Duflou, J., Yeates, L., Lam, L., .. , \& Semsarian, C. (2016). A Prospective Study of Sudden Cardiac Death among Children and YoungAdults. New England Journal of M edicine, 374(25), 2441-2452. doi:10.1056/ nejmoal510687

Baldi, E., Cornara, S., Contri, E., Epis, F., Fina, D. , Zelaschi, B., ... ., \& Somaschini, A. (2017). Real-time visual feedback during training improveslaypersons' CPR quality: arandomized controlled manikin study. Canadian Journal 
of Eemergency Medicine, 19(6), 480-7. https:/ / doi.org/ $10.1017 /$ cem. 2016.410

Basanta-Camiño, S., Navarro-Pđón, R., Freire-Tellado, M., Barcala-Furelos, R., Pavón-Prieto, M.P., Fernández López, M., \& NeiraPáaro, M.A. (2017). Evaluación del conocimiento y de las habilidades para el uso de un Desfibrilador Externo Automatizado (DEA) por estudiantes universitarios. Un diseño cuasiexperimenta. Medicina Intensiva, 41(5), 270-276. http:/ / dx.doi.org/ 10.1016/ j.medin.2016.07.008

Böttiger, B. W., Bossaert, L. L., Castrén, M., Cimpoesu, D., Georgiou, M., Greif, R. , ... \& M elieste, R. (2016). Kids Save Lives-ERC position statement on school children education in CPR: «Hands that help-Training children is training for life». Resuscitation, 105, A1-A3. https:/ / doi:10.1016/ j.resuscitation.2016.06.005.

Claesson, A., Svensson, L., Silfverstolpe, J., \& Herlitz, J. (2008). Characteristics and outcome among patients suffering out-of-hospital cardiac arrest dueto drowning Resuscitation, 76(3) 381-7. https:/ / doi.org/ 10.1016/ j.resuscitation.2007.09.003

Consejo Español deResucitación Cardiopulmonar (CERCP). (2012). Recomendaciones sobrelainstalación, autoriza ción y formación para el uso del desfibrilador externo automático fuera del ámbito sanitario [en línea]; 2012. Recuperado de http:/ / www. semicyuc.org/ sites/ d e f a u l t / f i l e s / recomendaciones_cercp_en_uso_dea_v. 0.9.pdf

Cortegiani,A., Russotto, V., M ontalto, F., Iozzo, P., M eschis, R., Pugliesi, M., ... , \& Giarratano, A. (2017). Use of a real-time training software (Laerdal QCPR $®$ ) compared to instructor-based feedback for high-quality chest compressions acqu. PLoS One, 12, 1-11. http:/ / dx. doi.org/ 10.1371/ journal. pone. 0169591

Gaintza, Z., \&Velasco, Z. (2020). Conocimiento del Profesorado de Infantil y Primaria en Reanimación Cardiopulmonar (Knowledge of Cardiopulmonary Resuscitation in Kindergarten and Primary School Teachers). Retos, N uevas tendencias en Educación Físca, de portey recreación, 39, 446-452. https./ / doi.org/ 10.47197/ retos. v0i39. 79354

González-Salvado,V., Fernández-M éndez. F., BarcalaFurelos, R., PeñaGil, C. , González-Juanđey, J.R., \& RodríguezNúñez, A. (2016). Very brief training for laypeople in hands-only cardiopulmonary resuscitation. Effect of realtime feedback. American Journal of Emergency Medidicine, 34 (6), 993-8. https:// doi.org/ 10.1016/ j.ajem. 2016.02.047

Hernández Padilla, J.M., Suthers, F., Granero M olina, J., \& Fernández Sola, C. (2015). Effects of two retraining strategies on nursing students' acquisition and retention of BLS/ AED skills: A cluster randomised trial. Resuscitation, 93, 27-34. 10.1016/ j.resuscitation. 2015.05.008.

Kelley J., Richman P.B., Ewy, G.A., Clark, L., Bulloch, B., $\&$ Bobrow B.J. (2006). Eighth grade students become proficient at CPR and use of an AED following a condensed ( training programme. Reuscitation, 71(2), 229236. 10.1016/ j.resuscitation.2006.03.015

Krasteva,V., IJekova, I., \& Didon, J.P. (2011). An audiovisual feedback device for compression depth, rate and complete chest recoil can improve the CPR performance of laypersonsduringself-trainingon amanikin. Physiol M eas, 32, 687-699 http:/ / dx.doi.org/ 10.1088/ 0967-3334/ 32/6/006

Lago-Ballesteros, J., BasantaCamiño, S., \& Navarro-Pđón, R. (2018). Laenseñanzadelosprimerosauxiliosen educación física: revisión sistemática acerca de los materia les para su implementación. Retos, N uevas tendencias en Educación Física, deportey recreación, 34, 349-355. https: / doi.org/ 10.47197/ retos. v0i34.65683

Ley O rgánica 8/ 2013, de 9 de diciembre, paraa mejora de la calidad educativa(Texto Consolidado). Boletín O ficial do Estado, 106, de 10 de diciembre de 2013. Recupera do de: https:/ / www. boe. es/ buscar/ pdf/ 2013/ BOEA-2013-12886-consolidado.pdf

Lukas, R.P., VanAken, H., Mölhoff,T., Weber, T., Rammert, M., W id, M., \& Bohn, A. (2016). Kids save lives: a sixyear longitudinal study of schoolchildren learning cardiopulmonary resuscitation: W ho should do the teaching and will the effects last? Resuscitation, 101, 3540. https:/ / doi: 10.1016/ j. resuscitation. 2016.01.028 Méndez-Martínez, C., Martínez-Isasi, S., García-Suarez, M., De la Peña-Rodríguez, M.A., Gómez-Salgado, J., \& Fernández-García, D. (2019). A cquisition of Knowledge and Practical Skills after a Brief Course of BLS AED in First Year Students in Nursing and Physiotherapy a aSpanish U niversity. International Journal of Environmental Research and Public Health, 16(5), 766. https:/ / doi.org/ 10.3390/ ijerph16050766

Morentin, B., Suárez-Mier, M.P., Aguilera, B. , Arrieta, J., Audicana, C., \& Fernández-Rodríguez, A. (2012). Clinicopathological features of sudden unexpected infectious death: Population-based study in children and young adults. Forensic Sá Int., 220, 80-4. Recuperado de http:/ / www. æeped.es/ sites/ default/ files/ documentos/ libro blanco muerte subita 3ed 1382521604.pdf Navarro, R., Penelas, G., \& Basanta, S. (2016). ¿Tienen las futuras maestras y maestros de educación primaria la formación necesaria para iniciar las maniobras de reani- 
mación cardiopulmonar en caso de emergencia escolar? Un estudio descriptivo. Educar, 52(1), 149-168. https: / / doi:10.5565/ rev/ educar. 714

Navarro-Patón, R., Cons-Ferreiro, M., M ecías-Calvo, M., \& Romo-Pérez,V. (2021). Acquisition of knowledge and skills on cardiopulmonary resuscitation and use of the automated external defibrillator after a training process by Galician schoolteachers. Journal of Human Sport and Exercise, in press. https:// doi.org/ 10.14198/ jhse. 2022.174.19

Navarro-Patón, R., Cons-Ferreiro, M., \& Romo-Pérez, V. (2020a). Conocimientos en soporte vital básico del profesorado gallego de educación infantil, primaria y secundaria: estudio transversal. Retos, N uevastendencias en Educación Físca, deportey recreación, 38, 173-179. https:/ I doi.org/ 10.47197/ retos. V38i38.75237

Navarro-Patón, R., Freire-Tellado, M., Basanta-Camiño, S. , BarcalaFfurelos, R.,Arufe-Girádez,V., \& RodríguezFernández, J. E. (2018). Efecto de 3 métodos de enseñanza en soporte vital básico en futuros maestros de educación primaria. un diseño cuasi-experimenta. Me diana Intensiva, 42 (4), 207-215. https:/ / doi:10.1016/ j.medin.2017.06.005

Navarro-Patón, R., Freire-Tellado, M., M ateos-Lorenzo, J., BasantaCamiño, S., Rodríguez-López, A., \& LagoBallesteros, J. (2020b) Sports Activities with Musical Support: A N ew Way to Learn Cardiopulmonary Resuscitation?Revista Internacional de Mediana y Ciencias delaActividad Físca y e D eporte20 (78) pp. 243-255. http:/ / doi.org/ 10.15366/ rimcafd2020.78.004

Navarro-Patón, R., Freire-Tellado, M., Pavón-Prieto, M.P., Vázquez-López, D., NeiraPájaro, M., \& Lorenzana Bargueiras, S. (2017). Dispatcher assisted Cardiopulmonary Resuscitation (CPR): is it important to continue teaching lay bystander CPR?. Am J Emerg Med. 35(4), 569-573. https:/ / dx. doi.org/ 10.1016/ j.ajem. 2016.12.014

Nishiyama, C., Iwami, T., Murakami, Y., Kitamura, T., O kamoto,Y., Marukawa, S., Sakamoto,T., \& Kawamura, T. (2015). Effectiveness of simplified 15-min refresher BLS training program: A randomized controlled trial. Resuscitation, 90, 56-60. http:/ / dx. doi.org/ 10.1016/ j.resuscitation. 2015.02.015

O lasveengen, T., M ancini, M.E., Perkins, G.D., Avis, S., Brooks, S., Maret Castrean, M., ... , \& Morley, P.T. (2020). Adult Basic Life Support 2020 International Consensus on Cardiopulmonary Resuscitation and Emergency Cardiovascular Care Science W ith Treatment Recommendations. Resuscitation, 166, A35A79. j. resuscitation. 2020.09.010

Pavón-Prieto M.P., Navarro-Patón R., Basanta-Camiño S., Regueira-Méndez C., Neira-Pájaro M.A., \& FreireTellado M. (2016) Estudio cuasi-experimental para evaluar la capacidad de los escolares para utilizar un desfibrilador externo semiautomático a los 6 meses tras un proceso formativo. Emergencias, 28, 114-6. https:/ / n9. $\mathrm{cl} / \mathrm{r} 6 \mathrm{j0q}$

Perkins, G. D., Handley, A. J., Koster, R. W., Castrén, M., Smyth, M. A., O lasveengen, T., ..., \& Ristagno, G. (2015). European Resuscitation Council Guidelines for Resuscitation 2015: Section 2. Adult basic life support and automated external defibrillation. Resuscitation, 95, 81-99. https:// doi.org/10.1016/ j. resuscitation. 2015.07.015

Riva, G., Ringh, M., Jonsson, M., Svensson, L., Herlitz, J., Claesson, A., ... , \& Hollenberg J. (2019). Survival in out-of-hospital cardiac arrest after standard Cardiopulmonary Resuscitation or chest compressions only before arrival of emergency medical services. Nationwide study during three guideline periods. Circulation, 139, 2600-9. https:/ / doi.org/ 10.1161/ CIRCULATIONAHA.118.038179

Sakaj-Bizmark, R., Friedlander, S. M. I., Marr, E. H., Mena, L. A., Corral, I., \& Chang, R.-K. R. (2018). Patient Characteristics and Emergency Department Factors Associated with Survival After Sudden Cardiac Arrest in Children andYoungAdults: A Cross-Sectional Analysis of a N ationally Representative Sample, 2006-2013. Pediatric Cardiology, 39 (6), 1216-1228. doi:10.1007/ s00246-018-1886-8

Semeraro, F., Frisoli, A., Loconsole, C., Mastronicola, N., Stroppa, F., Ristagno, G., ... , \& Cdrchiari, E. (2017). Kids (learn how to) save lives in the school with the serious game Relive. Resuscitation, 116, 27-32. https:/ / doi: 10.1016/ j.resuscitation.2017.04.038

Socias Crespí L., Ceniceros-Rozalen, M.I., Rubio-Roca, O., Martinez-Cuellar, N., García-Sánchez, A., Ripoll-Vera, T., \& Lesmes-Serrano, A. (2015). Características epidemiológicas de las paradas cardiorrespiratorias extrahospitalarias registradas por el sistema de emergencias 061 (SAMU) delaComunidadAutónoma de las Islas Baleares (2009-2012). M edicina Intensiva, 39, 199206. http:/ / dx.doi.org/ 10.1016/ j.medin.2014.04.004 